\title{
Slot-Ring Multiport Driven Antenna with Improved Airside Radiation for Terahertz Communications
}

\author{
Monageng Kgwadi, Khalid Alharbi, Jue Wang and Edward Wasige \\ High Frequency Electronics Group, School of Engineering, \\ University of Glasgow,G12 8QQ UK. \\ e-mail:m.kgwadi.1@research.gla.ac.uk: edward.wasige@glasgow.ac.uk
}

\begin{abstract}
A slot-ring multiport driven (MPD) antenna is evaluated for operation in the terahertz band with emphasis on air-side radiation on a thick Indium Phosphide (InP) substrate. Geometrical modification to the known slot-ring are made by employing a circular outer ground and a backing ground plane to focus the radiation in the airside direction. Optimization of the modified structure is made to find the optimal size of the upper ground plane. The optimal design achieves maximum directivity of $10.8 \mathrm{~dB}$ which is an improvement of $3 \mathrm{~dB}$ from the original design. Moreover, the radiation is directed in the airside direction with a front-to-back lobe ratio of $14 \mathrm{~dB}$. The slot-ring antenna design is verified by fabrication at $5 \mathrm{GHz}$ and achieves $10.2 \mathrm{~dB}$ directivity.
\end{abstract}

\section{INTRODUCTION}

The drive to accommodate the large number of devices envisaged in the Internet of Things (IoT) has led to the exploration of the terahertz $(300 \mathrm{GHz}-3 \mathrm{THz})$ band to open new communication channels. The terahertz band offers largely unregulated bandwidth for short-range high data-rate communications. As a result, a lot of work has been done by both industry and academia to develop terahertz communications [1], [2]. Resonant tunnelling diodes (RTDs) have been demonstrated to work as compact sources at room temperatures in the terahertz and sub-terahertz band [3]-[5]. RTD's therefore have the potential to make sub-Terahertz communications feasible if successfully coupled to antennas. The low power output of RTD-based oscillators $(<1 \mathrm{~mW})$ requires efficient antennas to achieve communication over longer distances (few meters). Planar antennas are favoured because of ease of manufacture and integration, but at the millimeter-wave frequencies they suffer from low efficiency due to the excitation of substrate modes as the substrate gets electrically larger than a quarter wavelength [6]. In most cases the thickness of the substrates can not be made thin due to manufacturing process and packaging constraints. The excitation of substrate modes confines some - and in some cases all [6]- of the antenna's radiated energy along the substrate instead of into the air. Moreover, the surface waves travelling along the substrate diffract at the edges as they exit the substrate, and interfere with the desired radiation pattern.

This study investigates a multiport driven (MPD) slot-ring antenna's feasibility to integrate with RTD-based oscillators. A brief introduction of MPD antennas and the slot-ring MPD antenna are introduced in Section II, outlining the current limitations. The geometry of the antenna was modified to improve air-side radiation of the slot-ring antenna. Further optimisation of the modified slot-ring antenna were made to achieve the optimal air-side radiation with minimum backside and lateral radiation. Conclusions and future work are presented in Section IV

\section{Multiport Driven Slot-Ring Antenna}

Multiport driven (MPD) radiators offer the possibility to obtain high radiation efficiency even with low input impedance antennas and power combining by the antenna [7]. An eight source multiport driven radiator designed for $155 \mathrm{GHz}$ operation implemented using a conductor ring with a wheel and spoke geometry on a quarter-wavelength thick silicon substrate with a reported gain of $4.4 \mathrm{dBi}, 9.71 \mathrm{dBi}$ maximum directivity and $30 \%$ efficiency was described in [7]. The key design parameter of an MPD ring antenna is $g=2 \pi r / \lambda$, the circumference to wavelength ratio of the ring, where $r$ is the radius of the loop. The design criteria is to create a loop with $1<g<\pi / 2$ in order to get the currents on the opposite halves of the loop and the center spokes to add up coherently and radiate effectively. The design in [7] features an multilayered structure with the spokes on a lower level and ring on a plane above the spokes, and two ground planes. A design challenge with the ring MPD antenna is to route the tracks to the driven points along the ring. A complementary structure of the loop antenna is a slot-ring and is presented in [8] which features integrated sources at desired points along the slot-ring. The slot-ring MPD in [8] was designed for backside radiation without the use of lenses due to the high dielectric constant of the substrate. The slot-ring is particularly interesting due to the relatively less complicated fabrication compared to the ring MPD requiring less metallisation layers. A drawback of the slot-ring is the backside radiation which we improve on in this study.

\section{A. $300 \mathrm{GHz}$ Slot-ring MPD}

A slot-ring antenna for $135 \mathrm{GHz}$ operation on a $250 \mu \mathrm{m}$ silicon substrate, $(0.41 \lambda)$ was presented in [8]. The effects of electrically thick susbstrate $(\geq 0.41 \lambda)$ on the slot-ring antenna are yet to be established and are investigated in this paper with the aim of establishing how much of an effect they have on the performance of the slot-ring MPD. To this effect, a $300 \mathrm{GHz}$ slot-ring MPD antenna was designed using the critea mentioned above and simulated in OpenEMS to 


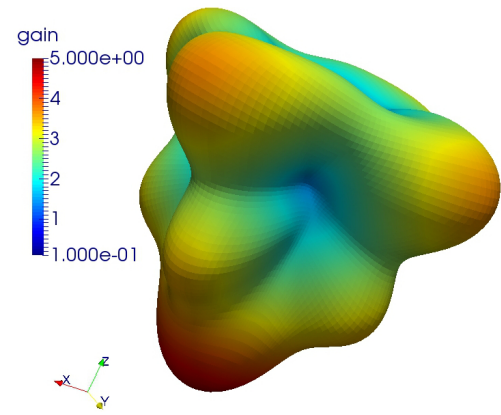

Fig. 1. Far field pattern of the slot-ring MPD on a 635 um thick substrate

investigate the effects of electrically thick substrates on the radiation pattern. OpenEMS is an open source finite-difference time-domain (FDTD) electro-magnetic field solver [9]. The designed slot ring has a radius of $45 \mu \mathrm{m}(g=1$ at $300 \mathrm{GHz})$, a slot aperture of $5 \mu \mathrm{m}$, while the substrate dimensions are $1000 \times 1000 \times 635 \mu \mathrm{m}$. Four sources separated by a quarter wavelength along the slot ring are driven with a quarter period delay (equivalent of 90 degree phase shift) from each other. Figure 1 shows the simulated directivity of the slotring with an electrically thick substrate. The pattern is on the backside of the substrate as is was the case in [7] but with significant lateral radiation. The electrically thick substrate supports more substrate modes which exit the substrate at the edges and cause lateral radiation which is also emphasized at the corners of the substrate. Figure 2 shows the evolution electric field distribution along the slot ring at successive time instances. The travelling wave along the slot is shown by the displacement of the peaks along the ring in a counterclockwise direction. The field intensities at the corners of the substrate (shown by dotted line) are high and we attribute them to the undesired lateral radiation shown in Figure 1 which ultimately reduce the directivity of the antenna.

\section{B. Airside Radiation Improvement}

The improvements we make on the initial design are to address 1) air-side radiation and 2) minimise lateral radiation at the edges of the substrate. We achieve this by adjusting the geometry of the outer ground plane to a circle and placing a ground plane on the backside of the substrate. The ring MPD presented in [7] uses a ground plane on the backside of the substrate but places a quarter wavelength substrate thickness requirement to guarantee coherent integration of the waves reflected from the ground for effective radiation. In this study a $2.25 \lambda$ (9 quarter wavelengths) thick substrate is used. Figure 3 shows the modified slot-ring MPD antenna. A circular outer ground plane minimizes the lateral radiation by allowing a purer circular travelling wave around the outer ground plane as shown in Figure 4 without corners which result in undesired radiation. The ground plane on the back of the substrate focuses the radiation on to the air side by reflecting waves that travel downwards. Figure 5 shows the directivity of the improved structure. There is an improvement on the directivity

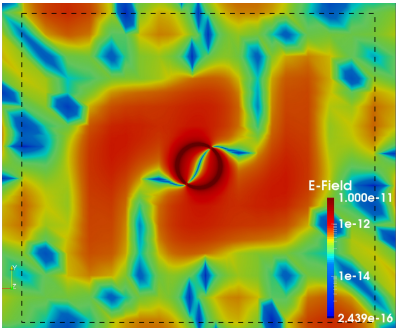

(a)

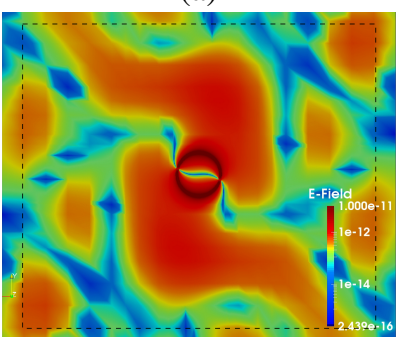

(c)

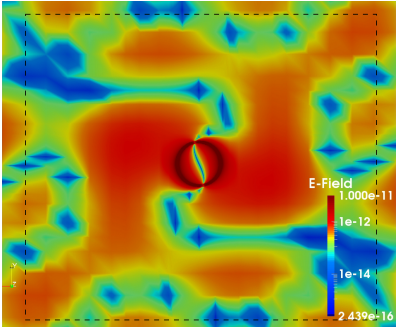

(e)

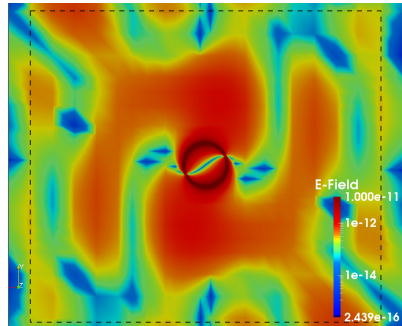

(b)

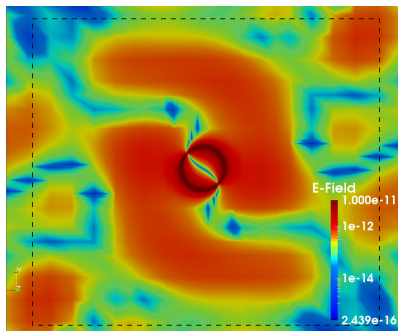

(d)

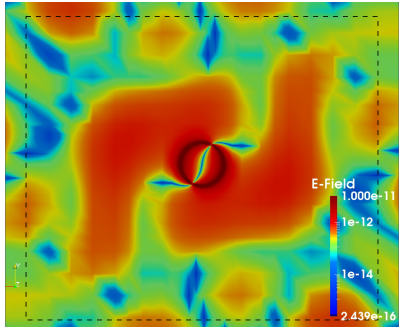

(f)
Fig. 2. Simulated electric field distribution showing the circularly polarised travelling wave within the slot at consecutive timesteps , (a) $t=0$, (b) $t=2$, (c) $t=4$ (d) $t=6$ (e) $t=8$, and (f) $t=10$.

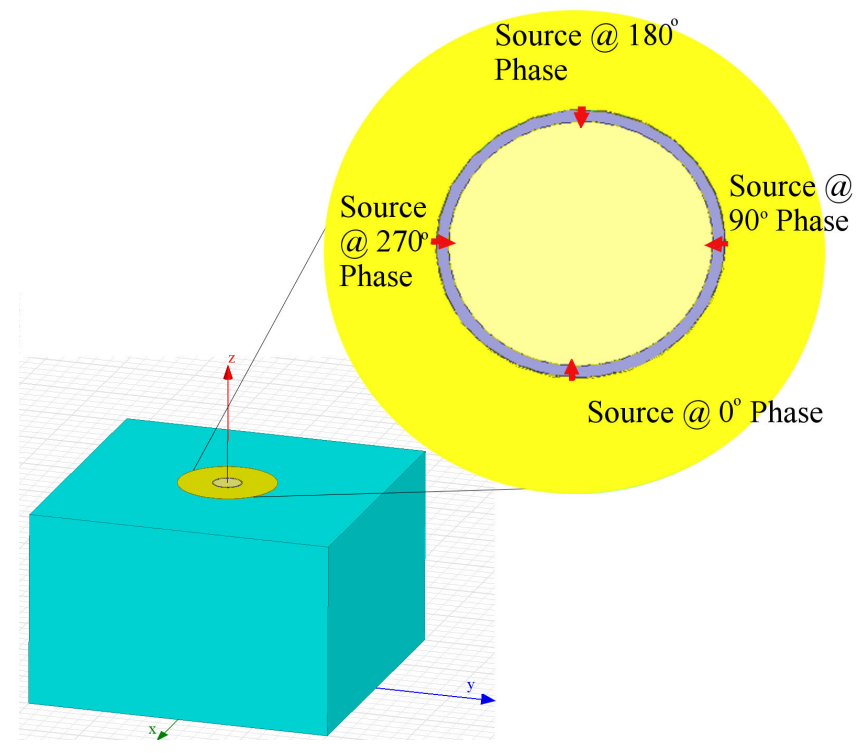

Fig. 3. MPD slot ring with backing ground plane for improved airside radiation

from $7.9 \mathrm{~dB}$ to $10.8 \mathrm{~dB}$ (approximately $3 \mathrm{~dB}$ increase) and the radiation is directed to the airside. 


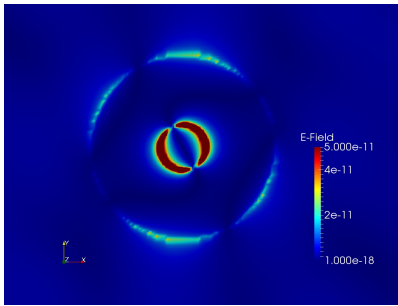

(a)

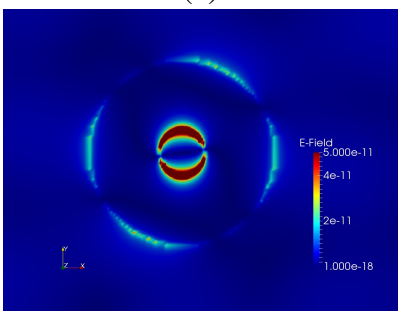

(c)

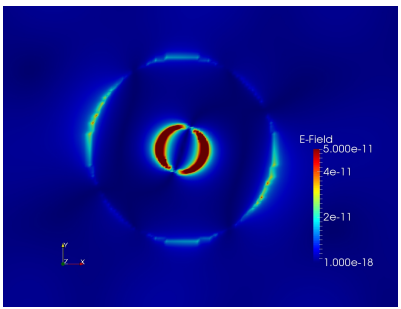

(e)

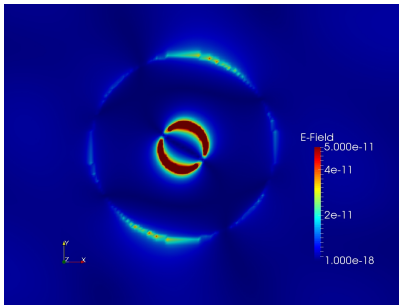

(b)

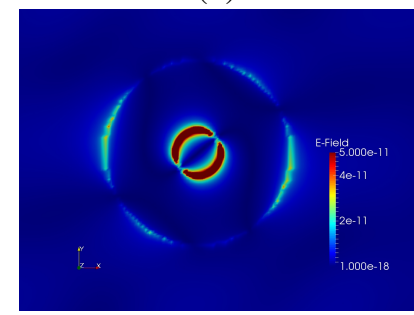

(d)

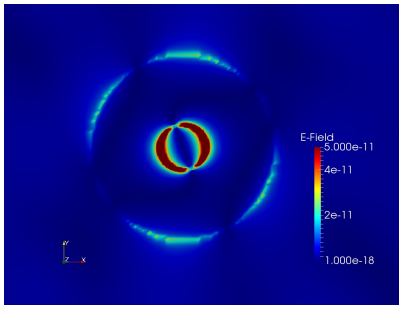

(f)
Fig. 4. Simulated electric field distribution showing the circularly polarised travelling wave within the modified slot-ring MPD at consecutive timesteps, (a) $\mathrm{t}=0$,(b) $\mathrm{t}=2$,(c) $\mathrm{t}=4$ (d) $\mathrm{t}=6$ (e) $\mathrm{t}=8$, and (f) $\mathrm{t}=10$.

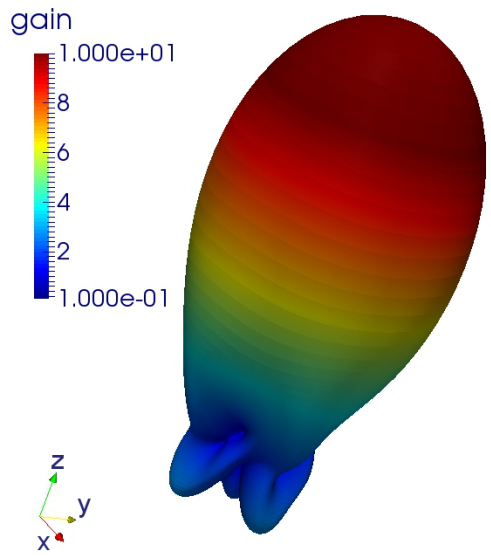

Fig. 5. Directivity of the improved slot-ring MPD on a $635 \mu \mathrm{m}$ thick substrate

\section{Optimization of Modified Slot-Ring MPD}

The initial results suggest that there should be an optimal size for the outer ground between the smallest value (thin loop surrounding the slot) to the maximum (covering the entire top surface). The geometry of the modified slot-ring MPD was varied to obtain the optimum directivity. The radius of the outer ground plane was varied keeping the size of the substrate constant at $1000 \times 1000 \times 635 \mu \mathrm{m}$ and the directivity was observed. Figure 6 shows the dependence of directivity on the size of the outer ground plane expressed in $H=2 \pi R_{g} / \lambda_{g}$, where $R_{g}$ is the radius of the outer ground plane and $\lambda_{g}$ is the guided wavelength. The directivity increases as the size of the outer ground plane is increased from the initial value of $8.60 \mathrm{dBi}$ at $\mathrm{H}=2$ and achieves a maximum value of $10.9 \mathrm{dBi}$ at $\mathrm{H}=4$ and reduces to a value of $6.4 \mathrm{dBi}$ at $\mathrm{H}=6$. This suggests that the optimum outer ground radius for maximum directivity is approximately $R_{g}=4 \lambda_{g} / 2 \pi$.

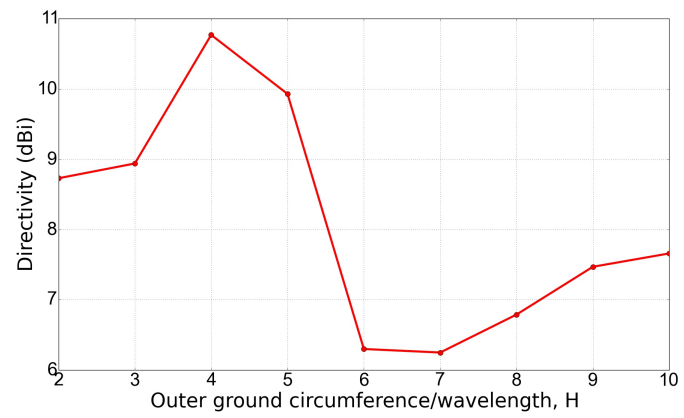

Fig. 6. Simulated maximum directivity versus the size of the outer ground plane

The front-to-back lobe ratio also shows a dependence on the size of the outer ground plane as shown by Figure 7. The modified slot-ring MPD shows a good front-to-back lobe ratio of between $13 \mathrm{dBi}$ and $14.8 \mathrm{dBi}$ in the range $H \in[2,5]$ beyond which a significant part of the radiation is directed to the back and laterally.

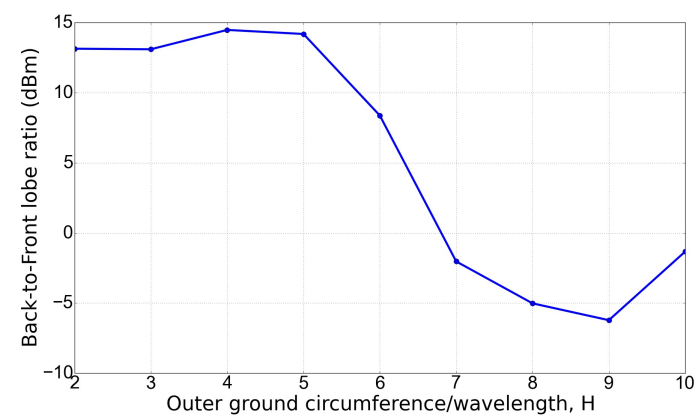

Fig. 7. Simulated front-to-back ratio versus the size of the outer ground plane

The input impedance at each port of the modified slotring antenna at the design frequency of $300 \mathrm{GHz}$ also shows a dependence on the size of ground plane as shown in Figure 8. The input impedance is inductive with the reactive part of the impedance between steadily increasing with $\mathrm{H}$ from $70 \Omega$ and $95 \Omega$ when $H \in[2,6]$ and reducing there after. The resistive part shows a relative steady value around $25 \Omega$ for $H \in[3,5]$, reaching a peak of $40 \Omega$ at $\mathrm{H}=6$ and then reducing to $11 \Omega$ for $\mathrm{H}$ greater than 9 .

\section{EXPERIMENTAL VALIDATION OF SLOT-RING ANTENNA}

A slot-ring antenna was designed and fabricated for $5 \mathrm{GHz}$ operation to validate the concept of a slot ring antenna with a 


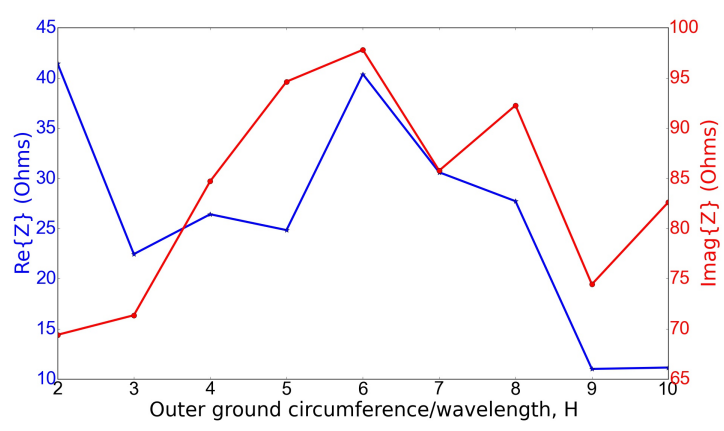

Fig. 8. Input impedance versus the ground size

backing ground plane. Figure 9 shows the fabricated antenna on $762 \mu \mathrm{m}$ of Rodgers laminated board $\left(\varepsilon_{r}=3.48\right)$ with $1 \mu \mathrm{m}$ thick copper. The dimensions were for maximum directivity of $\mathrm{H}=4$, with the outer circle radius of $25.41 \mathrm{~mm}$, slot radius, $r=7.57 \mathrm{~mm}$ and a slot width of $3 \mathrm{~mm}$ on a $100 \mathrm{~mm} \times 100 \mathrm{~mm}$ board. The antenna was fed at locations quarter wavelength apart and the phase difference was achieved by using two coax cables with a quarter wavelength difference in length. Figure 10 shows the simulated and measured far field radiation pattern. The measured patterns matches simulated pattern with an unexpected null attributed to the coax cables that run across the front of the antenna. Feeding the antenna from the back through the use of narrower cables is expected to eliminate the effects of the cables. The measured directivity is $10.2 \mathrm{~dB}$ which is similar to the simulated value of $10.8 \mathrm{~dB}$.

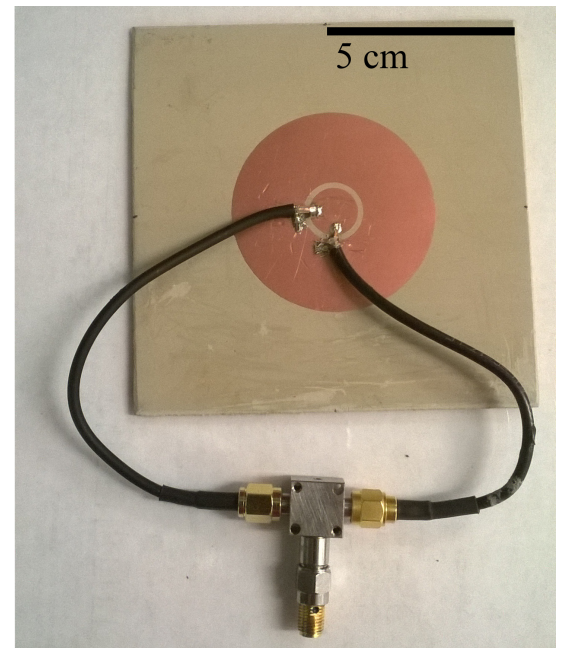

Fig. 9. Fabricated slot ring antenna for $5 \mathrm{GHz}$ operation

\section{CONCLUSION}

We present an improved slot-ring multi-port driven antenna with air-side radiation for operation at $300 \mathrm{GHz}$. An optimal design for the improved antenna is also achieved along with relations of the directivity and input impedance on the geometry of the antenna. A maximum simulated directivity of

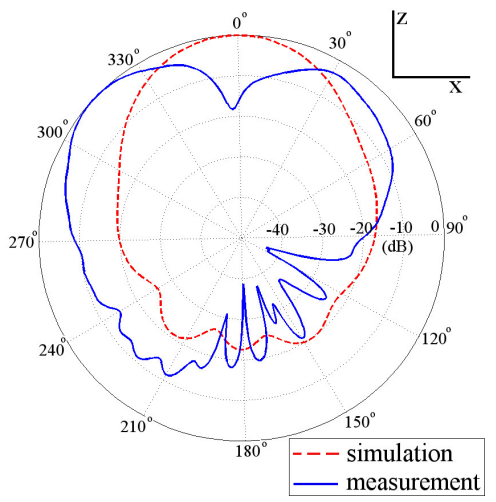

Fig. 10. Simulated and measured normalised far-field radiation pattern of the $5 \mathrm{GHz}$ slot ring antenna

the improved slot-ring MPD antenna is $10.8 \mathrm{~dB}$ with a sideto-back lobe ratio of $14.8 \mathrm{~dB}$. The concept of the slot-ring was experimentally verified by a fabricated antenna for $5 \mathrm{GHz}$ operation showing the expected performance. Future work involves designing an integrated driving circuit, fabrication and measurement of the performance of the system

\section{ACKNOWLEDGMENT}

This work was supported in part by the European Commission, grant agreement no. 645369 (iBROW Project)

\section{REFERENCES}

[1] D. Britz, "Wireless big data are our wireless edge networks ready?" in International Conference on Infrared, Millimeter, and Terahertz waves (IRMMW-THz), Sept 2014, pp. 1-4.

[2] I. Hosako, N. Sekine, M. Patrashin, S. Saito, K. Fukunaga, Y. Kasai, P. Baron, T. Seta, J. Mendrok, S. Ochiai, and H. Yasuda, "At the dawn of a new era in terahertz technology," Proceedings of the IEEE, vol. 95, no. 8, pp. 1611-1623, Aug 2007.

[3] K. Okada, K. Kasagi, N. Oshima, S. Suzuki, and M. Asada, "Resonanttunneling-diode terahertz oscillator using patch antenna," IEEE Transactions on Terahertz Science and Technology,.

[4] A. Suminokura, K. Tsuruda, T. Mukai, M. Fujita, and T. Nagatsuma, "Integration of resonant tunneling diode with terahertz photonic-crystal waveguide and its application to gigabit terahertz-wave communications," in International Topical Meeting on Microwave Photonics (MWP) and the 9th Asia-Pacific Microwave Photonics Conference (APMP), Oct 2014, pp. 419-422.

[5] J. Wang, K. Alharbi, A. Ofiare, H. Zhou, A. Khalid, D. Cumming, and E. Wasige, "High performance resonant tunneling diode oscillators for thz applications," in 2015 IEEE Compound Semiconductor Integrated Circuit Symposium (CSICS), Oct 2015, pp. 1-4.

[6] A. Shahvarpour, A. Alvarez Melcon, and C. Caloz, "Radiation efficiency issues in planar antennas on electrically thick substrates and solutions," IEEE Transactions on Antennas and Propagation, vol. 61, no. 8, pp. 4013-4025, Aug 2013.

[7] S. Bowers and A. Hajimiri, "Multi-port driven radiators," IEEE Transactions on Microwave Theory and Techniques, , vol. 61, no. 12, pp. 44284441, Dec 2013.

[8] S. Bowers, A. Safaripour, and A. Hajimiri, "An integrated traveling-wave slot radiator," in IEEE Radio Frequency Integrated Circuits Symposium, June 2014, pp. 369-372.

[9] T. Liebig. openems - open electromagnetic field solver. General and Theoretical Electrical Engineering (ATE), University of Duisburg-Essen. [Online]. Available: http://openEMS.de 